\title{
SIMULAÇÃO DE CICLOS DE POTÊNCIA E REFRIGERAÇÃO PARA O PROCESSO DE LIQUEFAÇÃO DO GÁS NATURAL
}

\author{
L. V. Moura ${ }^{1}$ e J. V. H. D’Angelo ${ }^{1}$ \\ ${ }^{1}$ Universidade Estadual de Campinas, Faculdade de Engenharia Química \\ E-mails para contato: dangelo@,feq.unicamp.br, leonardovilarm@gmail.com
}

\begin{abstract}
RESUMO - O gás natural, mistura de hidrocarbonetos constituída essencialmente de metano, representa uma alternativa de combustível perante aos demais combustíveis fósseis, dada a sua baixa emissão de gases poluentes amplificadores do efeito estufa, tais como $\mathrm{SO}_{2}, \mathrm{H}_{2} \mathrm{~S}, \mathrm{NO}_{\mathrm{x}}$ e, também, custo reduzido. Entretanto, o transporte do gás natural para longas distâncias requer que o mesmo se encontre a um volume reduzido, principalmente para tornar este transporte viável economicamente. O GNL (gás natural liquefeito) é obtido a partir de processos industriais que demandam grande quantidade de energia. A otimização e proposição de melhorias desses processos leva a ganhos significativos no coeficiente de desempenho dos ciclos responsáveis pela liquefação do gás natural, diminuindo o custo de operação dessas plantas químicas. Neste trabalho, foi utilizado o software Aspen Hysys ${ }^{\circledR}$ v7.3 para simular ciclos de potência e de refrigeração, utilizados no processo de liquefação do gás natural, determinando sua eficiência termodinâmica e quantificando a energia elétrica necessária para seu funcionamento e posterior otimização para uma combinação de ciclos.
\end{abstract}

\section{INTRODUÇÃO}

Embora a busca por fontes de energia renováveis venha crescendo intensamente, ainda é necessária a utilização de fontes de energia não-renováveis para atender à demanda energética em nível mundial. Neste contexto, os combustíveis fósseis são amplamente empregados pela indústria em diversos processos químicos. O gás natural, derivado mais limpo dos combustíveis fósseis, apresenta alta eficiência energética de queima, a um custo baixo, tornando-se então um importante combustível e também matéria-prima em muitos processos químicos industriais. Para contextualizar a situação atual do país, segundo Boletim da Produção de Petróleo e Gás Natural da Agência Nacional do Petróleo, Gás Natural e Biocombustíveis (ANP), a produção diária do gás natural em fevereiro de 2014 foi de aproximadamente $83,2 \mathrm{MMm}^{3} /$ dia (milhões de metros cúbicos por dia) e apresentou um aumento de $8,8 \%$ na sua produção em relação ao mesmo mês do ano anterior.

O volume ocupado pelo gás natural em sua forma liquefeita (GNL) é cerca de 600 vezes menor em relação à forma gasosa, daí a grande necessidade em liquefazer o gás natural com intuito de viabilizar o seu transporte a longas distâncias. Entretanto, processos capazes de levar o gás natural a temperaturas próximas de $-160^{\circ} \mathrm{C}$ à pressão atmosférica consomem 
aproximadamente $1188 \mathrm{~kJ}$ de energia para cada $1 \mathrm{~kg}$ de gás natural (Finn et al., 1999), dependendo do ciclo de refrigeração (liquefação) utilizado no processo. Os principais equipamentos responsáveis pelo consumo de energia são os compressores presentes no ciclo de refrigeração (C.R.), os quais são acionados por meio da energia elétrica produzida em um ciclo de potência (C.P.), atuando de forma conjunta ao processo de liquefação do gás natural.

Devido a esta dependência do consumo de energia no processo de liquefação do gás natural, é essencial analisar e determinar condições de operação para variáveis como temperatura, pressão, vazão, fração de vapor, etc; para produzir ciclos com melhores rendimentos e, consequentemente, menores custos.

Neste sentido, o objetivo deste trabalho foi simular ciclos de refrigeração e potência, utilizados no processo de produção de GNL, com base em artigos retirados da literatura, para analisar a influência das variáveis de processo destes ciclos sobre seu desempenho termodinâmico, visando a otimização dos mesmos. Para a execução deste projeto foi escolhido o simulador Aspen Hysys ${ }^{\circledR}$ v7.3, que além da análise termodinâmica da eficiência dos ciclos permitiu também realizar a otimização de uma única combinação de ciclos em busca de melhor desempenho e eficiência.

\section{METODOLOGIA}

Os processos responsáveis pela liquefação do gás natural podem variar de acordo com a capacidade produtiva desejada da planta, tipos de ciclo de refrigeração, composição e vazão do fluido refrigerante, quantidade de compressores, entre outros. Neste trabalho, foram adotados três ciclos de refrigeração e três ciclos de potência para produção de gás natural liquefeito e, ao longo do trabalho, os mesmos serão reproduzidos e será feita uma análise de suas configurações ótimas visando o aumento da eficiência e coeficiente de desempenho para apenas uma combinação de ciclos, isto é, um Ciclo de Potência e um Ciclo de Refrigeração. Tais etapas, incluindo a otimização de ambos os ciclos, será feita no simulador de processos Aspen Hysys ${ }^{\circledR}$ v7.3.

O uso de simuladores de processo é muito comum tanto na indústria quanto no ambiente acadêmico como forma de validar modelos de processos, analisar interferência e sensibilidade de variáveis como temperatura, pressão, vazão e, também, propor novas alternativas aos processos, sem o custo de um investimento em larga escala.

Eficiência Termodinâmica: A eficiência térmica $\eta$ de um ciclo de potência, pode ser calculada, de maneira genérica, pela Equação 1.

$$
\eta=\frac{W_{t}-W_{b}}{Q}
$$

Em que $W_{\mathrm{t}}$ é o trabalho produzido pelas turbinas, $W_{\mathrm{b}}$ o trabalho consumido pelas bombas e $Q$ o calor fornecido na caldeira pela queima de um combustível.

Coeficiente de Desempenho (COP): Para cálculo do COP de um ciclo de refrigeração, é necessário saber a carga térmica $Q$ dos trocadores de calor (LNG Exchanger) e também a 


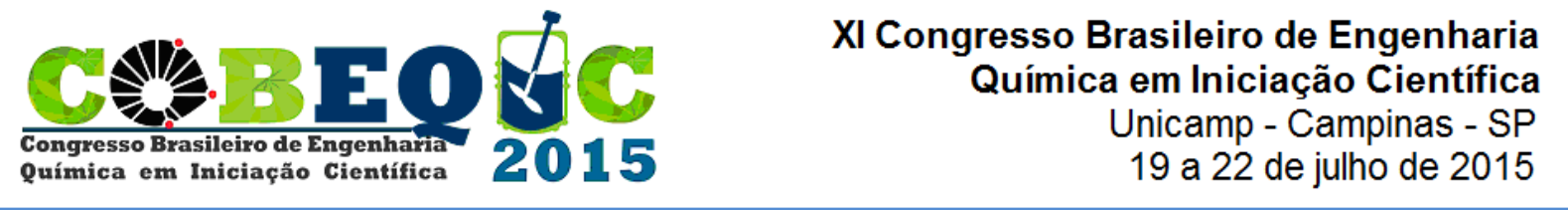

quantidade total de potência necessária nos compressores $\mathrm{W}_{\text {comp. }}$. Dessa forma, o COP é dado pela Equação 2.

$$
C O P=\frac{Q}{W_{\text {comp }}}
$$

\section{RESULTADOS E DISCUSSÃO}

Nesta seção são apresentados os principais resultados referentes às simulações feitas no Aspen Hysys ${ }^{\circledR}$ v7.3 dos ciclos de potência e refrigeração encontrados em artigos na literatura.

\subsection{Ciclos de Potência}

Primeiramente, foram simulados, a partir de artigos retirados da literatura, três ciclos de potência. Os resultados foram satisfatórios e apresentaram, em média, desvios inferiores a 5\% em relação aos dados da literatura. Os modelos validados, poderão ser posteriormente utilizados para fornecer a potência necessária aos compressores dos ciclos de refrigeração.

O Ciclo de Potência I (Gebreegziabher et al., 2014) utiliza a água como fluido de trabalho a uma vazão de $35287 \mathrm{~kg} / \mathrm{h}$ e é composto, essencialmente, de três turbinas conectadas em série, todas com eficiência isentrópica de $80 \%$; três bombas com eficiências supostas igual a 100\%; uma caldeira; misturadores e separadores. Os resultados obtidos na simulação apresentaram discrepâncias menores que 1\% em relação a Gebreegziabher et al. (2014), com exceção apenas na corrente 2 do processo, onde foi encontrado o valor de 0,94 para a Fração de Vapor, ao invés de 0,96. Pequenas diferenças encontradas se dão, em grande parte, devido à utilização de simuladores e equações de estado diferentes.

Para o Ciclo I, mostrado na Figura 1, a eficiência termodinâmica obtida a partir da Equação 1 foi de $\eta=0,3660$, valor considerado aceitável e próximo dos valores trabalhados em ciclos de potência que utilizam água como fluido de trabalho (Çengel e Boles, 2001).

Figura 1: Fluxograma do Ciclo de Potência I no Aspen Hysys ${ }^{\circledR}$ v7.3.

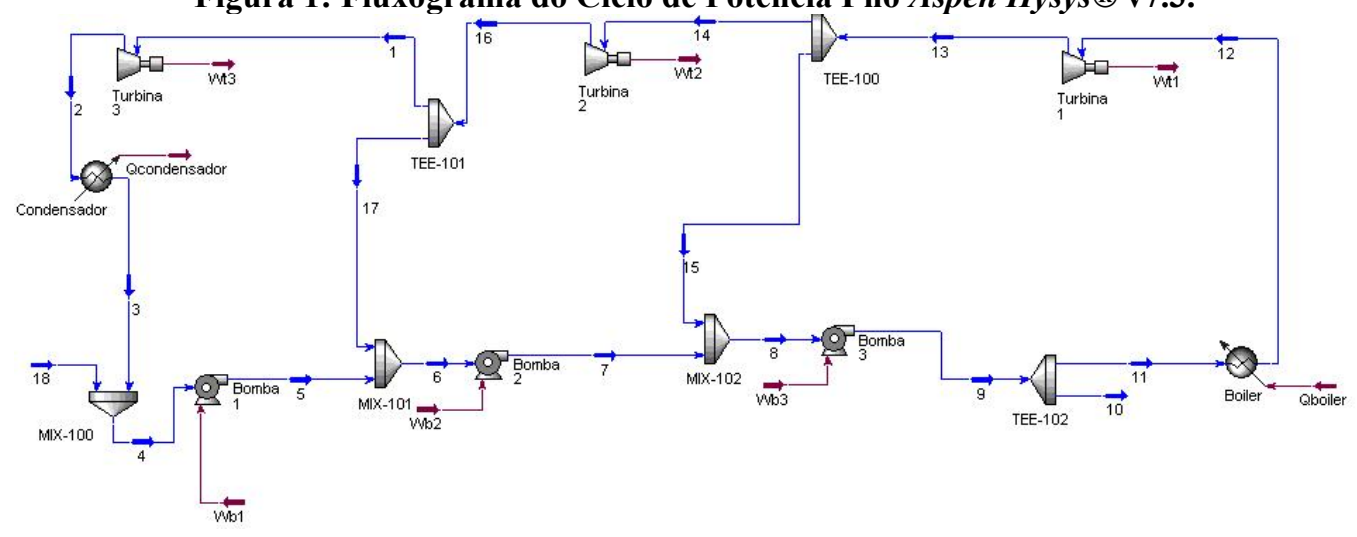

A Tabela 1 mostra que os dados obtidos a partir da reprodução do artigo no simulador de processos são satisfatórios, pois apresentaram pequenos desvios em relação ao artigo de referência. Desta maneira, pode-se considerar a simulação satisfatória. 
Tabela 1 - Dados simulados e dados da literatura para o ciclo da Figura 1.

\begin{tabular}{cccccc}
\hline Temperatura $\left({ }^{\circ} \mathrm{C}\right)$ & Corrente 1 & Corrente 2 & Corrente 4 & Corrente 6 & Corrente 8 \\
\hline Simulação & 207,70 & 45,83 & 40,82 & 56,87 & 126,80 \\
Referência $^{*}$ & 207,85 & 45,85 & 40,85 & 56,85 & 126,85 \\
\hline
\end{tabular}

*Gebreegziabher et al. (2014)

A Tabela 2 contém informações básicas referentes aos três ciclos de potência reproduzidos no simulador. É importante observar que, dados como eficiência térmica e potência gerada $(W)$ são de grande utilidade para cálculos futuros envolvendo a associação entre os ciclos de potência e refrigeração e suas condições ótimas de operação.

Tabela 2 - Dados dos ciclos de potência.

\begin{tabular}{cccccc}
\hline Ciclo & Referência & $\begin{array}{c}\text { Vazão } \\
(\mathrm{kg} / \mathrm{h})\end{array}$ & $\begin{array}{c}\text { Fluido de } \\
\text { Trabalho }\end{array}$ & $\begin{array}{c}\text { Eficiência } \\
(\eta)\end{array}$ & $\begin{array}{c}W \\
\left(10^{7} \mathrm{~kJ} / \mathrm{h}\right)\end{array}$ \\
\hline I & Gebreegziabher et al. (2014) & 35287,0 & $\mathrm{H}_{2} \mathrm{O}$ & 0,3660 & 4,67 \\
II & Holmberg e Ahtila (2014) & 69192,0 & $\mathrm{H}_{2} \mathrm{O}$ & 0,3116 & 6,19 \\
III & Shi e Che (2009) & 86060,0 & $\mathrm{H}_{2} \mathrm{O}$ e NH $\mathrm{NH}_{3}$ & 0,3385 & 3,09 \\
\hline
\end{tabular}

\subsection{Ciclos de Refrigeração}

Em todos os casos, a equação de estado utilizada pelo simulador para cálculo das propriedades termodinâmicas é a de Peng-Robinson, por apresentar resultados satisfatórios para hidrocarbonetos e substâncias apolares em geral. Assim como no Ciclo de Refrigeração I, mostrado na Figura 2, priorizou-se nos demais ciclos a proximidade dos valores de temperatura das correntes entre a simulação feita e o respectivo artigo de referência, tendo como consequência maiores discrepâncias encontradas nos valores de pressão.

Figura 2: Fluxograma do Ciclo de Refrigeração I no Aspen Hysys ${ }^{\circledR}$ v7.3.

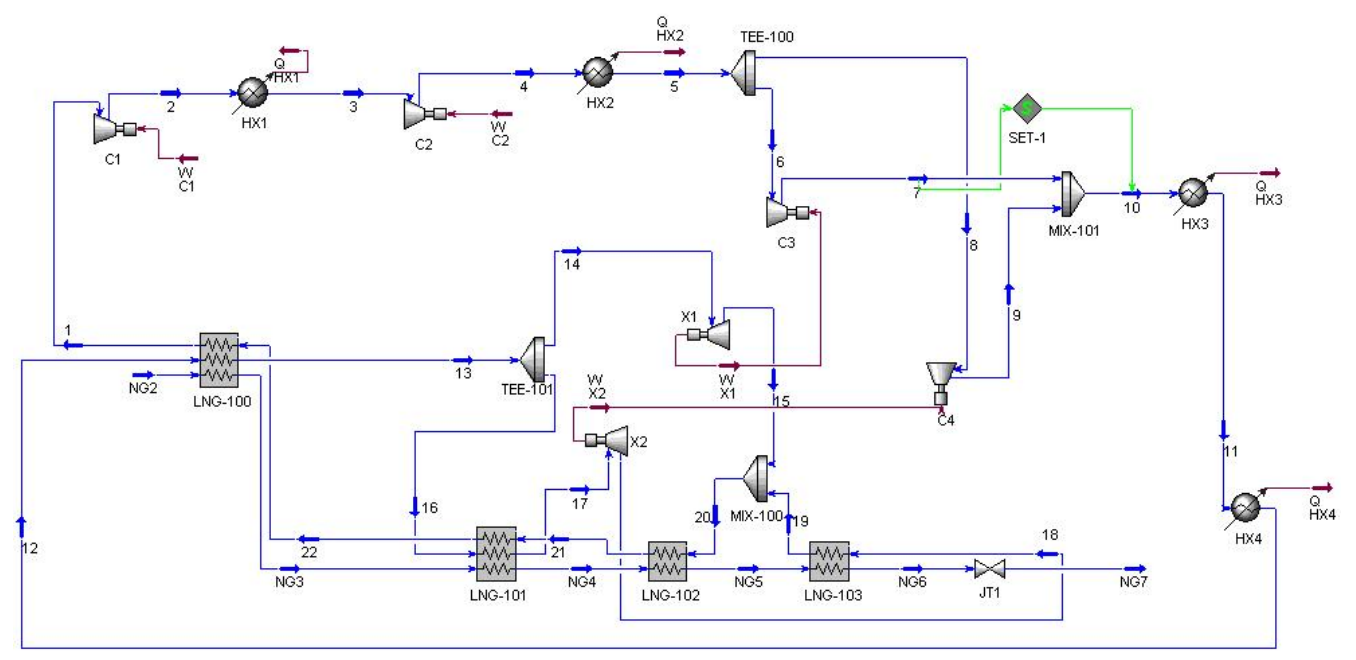

Em suma, os resultados obtidos para os três Ciclos de Refrigeração, tal como a potência total requerida $W_{\text {comp }}$, podem ser vistos na Tabela 3 . 
Tabela 3 - Dados dos Ciclos de Refrigeração.

\begin{tabular}{cccccc}
\hline Ciclo & Referência & $\begin{array}{c}\text { Vazão GNL } \\
(\mathrm{kg} / \mathrm{h})\end{array}$ & Refrigerante & COP & $\begin{array}{c}\mathrm{W}_{\text {comp }} \\
\left(10^{7} \mathrm{~kJ} / \mathrm{h}\right)\end{array}$ \\
\hline I & Song et al. $(2014)$ & 82718,0 & $\mathrm{~N}_{2}$ & 0,2907 & 22,500 \\
II & Yuan et al. $(2014)$ & 58,9 & $\mathrm{~N}_{2} \mathrm{e} \mathrm{CO}_{2}$ & 0,7680 & 0,015 \\
III & Chang et al. $(2011)$ & 3600,0 & $\mathrm{~N}_{2}, \mathrm{C}_{2} \mathrm{H}_{6} \mathrm{e} \mathrm{C}_{3} \mathrm{H}_{8}$ & 0,4210 & 0,673 \\
\hline
\end{tabular}

\subsection{Otimização}

A otimização feita neste trabalho baseou-se no acoplamento de ambos os Ciclos I de Potência e Refrigeração, descritos anteriormente e para tal foi utilizada uma ferramenta de otimização do próprio simulador Aspen Hysys ${ }^{\circledR}$ v7.3, o Optimizer (método MIXED). Esta ferramenta buscar minimizar ou maximizar uma dada função objetivo, por meio da manipulação de variáveis como temperatura, pressão, fração de vapor, em determinadas faixas pré-escolhidas. No caso dos ciclos de potência e refrigeração, avaliados separadamente, buscou-se a maximização da eficiência termodinâmica e a minimização da potência necessária nos compressores, respectivamente. A equivalência entre a potência necessária no ciclo de refrigeração e a produzida no ciclo de potência foi atingida por meio da função Adjust do simulador, utilizando a vazão mássica de água como variável ajustável. Os resultados, que incluem as variáveis de cada ciclo que apresentaram maior alteração após a otimização, podem ser vistos na Tabela 4.

A minimização do $\mathrm{W}_{\text {comp }}$ feita pelo Optimizer resultou, diretamente, em um aumento significativo do COP, cerca de 54\%, elevando-o ao valor de 0,4481. Durante este processo de otimização, no ciclo de refrigeração, a vazão de refrigerante foi a variável que apresentou a maior alteração, partindo de $1,3 \times 10^{6} \mathrm{~kg} / \mathrm{h}$ para $0,9 \times 10^{6} \mathrm{~kg} / \mathrm{h}$. Já a otimizição realizada no ciclo de potência, teve a pressão da corrente 13 como a variável que sofreu maior alteração, isto é, uma variação de $800 \mathrm{kPa}$. Para este caso, a maximização da Eficiência Termdinâmica, porém, resultou em um aumento de 0,3660 para 0,3677 .

Tabela 4 - Resultados antes e depois da otimização (Optimizer).

\begin{tabular}{|c|c|c|c|c|c|c|}
\hline & $\begin{array}{c}\text { Eficiência } \\
\text { Termodinâmica } \\
(\eta)\end{array}$ & COP & $\begin{array}{c}\mathrm{W}_{\text {comp }} \\
\left(10^{7} \mathrm{~kJ} / \mathrm{h}\right)\end{array}$ & $\begin{array}{l}\text { Pressão } \\
(\mathrm{kPa}) \\
\text { Corrente } \\
13 \text { (C.P.) }\end{array}$ & $\begin{array}{c}\text { Vazão de } \\
\text { Refrigerante } \\
\left(10^{6} \mathrm{~kg} / \mathrm{h}\right)\end{array}$ & $\begin{array}{c}\text { Vazão de } \\
\mathrm{H}_{2} \mathrm{O} \\
(\mathrm{kg} / \mathrm{h})\end{array}$ \\
\hline Antes & 0,3660 & 0,2907 & 22,5 & 1800,0 & 1,3 & 35287,0 \\
\hline Depois & 0,3677 & 0,4481 & 14,6 & 1000,0 & 0,9 & 126700,0 \\
\hline
\end{tabular}

\section{CONCLUSÃO}

Neste trabalho, buscou-se avaliar a importância e necessidade de melhorias em processos de liquefação do gás natural, utilizando um simulador de processos para realizar uma análise da influência de algumas variáveis operacionais sobre o desempenho 


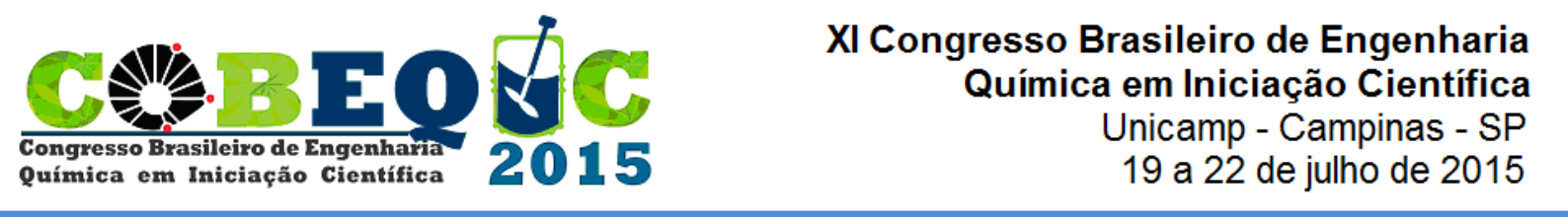

termodinâmico dos ciclos envolvidos no processo de liquefação. Os ciclos de potência e refrigeração adotados e reproduzidos por meio do simulador Aspen Hysys ${ }^{\circledR}$ v7.3 apresentaram bons resultados em relação aos artigos de referência utilizados, permitindo validar os modelos construídos no simulador. A otimização de uma combinação de Ciclos de Potência e Refrigeração, por meio da função Optimizer, resultou em uma diminuição significativa no valor da potência requerida pelos compressores e, consequentemente, levou a um aumento do COP do ciclo. Embora a otimização do ciclo de potência não tenha acarretado em grandes alterações na eficiência termodinâmica, sua importância tornou-se visível ao permitir que o aumento da vazão de $\mathrm{H}_{2} \mathrm{O}$ resultasse na produção de energia elétrica necessária para a ativação dos compressores.

\section{REFERÊNCIAS}

ÇENGEL, Y. A.; BOLES, MICHAEL A.

Thermodynamics: an engineering approach. : McGraw-Hill. p. 588, 2001.

CHANG, H. M.; CHUNG, M. J.; LEE, S.; CHOE, K. H.

An efficient multi-stage Brayton-JT cycle for liquefaction of natural gas. Cryogenics., v. 51(6), p. 278-286, 2011.

FINN, A. J.; JOHNSON, G. L.; TOMLINSON, T. R.

Developments in natural gas liquefaction. Hydrocarbon Processing., v. 78(4), p. 47-59, 1999.

GEBREEGZIABHER, T.; OYEDUN, A. O.; LUK, H. T.; LAM, T. Y. G.; ZHANG, Y.; HUI, C. W. Design and optimization of biomass power plant. Chemical Engineering Research and Design., v. 92(8), p. 1412-1427, 2014.

HOLMBERG, H.; AHTILA, P.

The thermal analysis of a combined heat and power plant undergoing Clausius-Rankine cycle based on the theory of effective heat-absorbing and heat-emitting temperatures. Applied Thermal Engineering., v. 70(1), p. 977-987, 2014.

SHI, X.; CHE, D.

A combined power cycle utilizing low-temperature waste heat and LNG cold energy. Energy Conversion and Management., v. 50(3), p. 567-575, 2009.

SONG, K.; LEE, S.; SHIN, S.; LEE, H. J.; HAN, C.

Simulation-based optimization methodology for offshore natural gas liquefaction process design. Industrial and Engineering Chemistry Research., v. 53(13), p. 55395544, 2014.

YUAN, Z.; CUI, M.; XIE, Y.; LI, C.

Design and analysis of a small-scale natural gas liquefaction process adopting single nitrogen expansion with carbon dioxide pre-cooling. Applied Thermal Engineering., v. 64(1-2), p. 139-146, 2014. 